\title{
Growth mindset is not associated with scholastic aptitude in a large sample of university applicants
}

\author{
Štěpán Bahník ${ }^{1}$ and Marek A. Vranka $a^{2,3}$ \\ ${ }^{1}$ Faculty of Business Administration, University of Economics, Prague, náměstí Winstona \\ Churchilla 4, Prague, 130 67, Czech Republic. bahniks@seznam.cz \\ ${ }^{2}$ Faculty of Social Sciences, Charles University, Smetanovo nábřeží 6, Prague, 110 01, Czech \\ Republic. marek.vranka@fsv.cuni.cz \\ ${ }^{3}$ Faculty of Arts, Charles University, nám. J. Palacha 2, Prague, 116 38, Czech Republic. \\ marek.vranka@ff.cuni.cz
}

\begin{abstract}
Implicit theories of intelligence have been proposed to predict a large number of different outcomes in education. The belief that intelligence is malleable (growth mindset) is supposed to lead to better academic achievement and students' mindset is therefore a potential target for interventions. The present study used a large sample of university applicants $(N=5,653)$ taking a scholastic aptitude test to further examine the relationship between mindset and achievement in the academic domain. We found that results in the test were slightly negatively associated with growth mindset $(r=-.03)$. Mindset showed no relationship with the number of test administrations participants signed up for and it did not predict change in the test results. The results show that the strength of the association between academic achievement and mindset might be weaker than previously thought.
\end{abstract}

\section{Keywords}

mindset, scholastic aptitude, achievement, implicit theories, education, intelligence, selfregulation, performance

Intelligence predicts a large number of important life outcomes (Neisser et al., 1996). Apart from the level of intelligence itself, people may also differ in their implicit theories about the nature of intelligence and its malleability. On one end of the continuum, some people consider intelligence fixed and unchangeable. On the opposite side, some people believe that intelligence can be improved and developed. These two views are labeled as a fixed mindset and growth mindset, respectively (Dweck, 2012; Dweck \& Leggett, 1988; Molden \& Dweck, 2006). A recent metaanalysis showed that implicit theories about intelligence are associated with various aspects of self-regulation (Burnette, O'Boyle, VanEpps, Pollack, \& Finkel, 2013). People with growth 
mindset tend to set learning goals rather than performance goals, they tend to adopt strategies focused on mastery rather than helpless-oriented strategies, and when monitoring their goal achievement, they tend to focus on future expectations of success rather than negative emotions. Importantly, the self-regulatory processes associated with growth mindset are positively associated with goal achievement. Growth mindset itself was therefore shown to be associated with a higher likelihood of achieving one's goals; even though, the results of the meta-analysis suggest that the association is weak (Burnette et al., 2013).

While Burnette et al. (2013) showed the association between implicit theories and achievement, some research (e.g., Blackwell, Trzesniewski, \& Dweck, 2007; see also Yeager et al., 2014) suggests that people with growth and fixed mindset may not differ in their baseline abilities and the difference appears only when encountering adversities or challenging situations. For example, students with growth and fixed mindset may not differ in their mathematics grades before entering junior high school, but the transition to high school poses a significant challenge to which students with growth mindset might adapt better. As a results, mathematics grades were shown to start to diverge between students with growth and fixed mindset over the first two years of junior high school; even though, they were at a similar level at the beginning of high school (Blackwell et al., 2007).

Given the association of growth mindset with goal achievement, changing students' mindsets has been proposed as a possible intervention for improving academic achievement (Rattan, Savani, Chugh, \& Dweck, 2015). Supporting this possibility, some studies demonstrated that interventions teaching the malleability view of intelligence positively affected academic achievement among high school (e.g., Paunesku et al., 2015; Yeager et al., 2016a) as well as university students (Yeager et al., 2016b).

The present study explores the association between implicit theories of intelligence and results of a scholastic aptitude test in a large sample of 5,653 university applicants. A number of previous studies show that results in similar tests of scholastic aptitude can be improved by general or special preparation (e.g., Becker, 1990; Montgomery \& Lilly, 2012; Powers, 1985; Powers \& Rock, 1999), which suggests that self-regulatory processes associated with growth mindset might positively influence the performance on the test, assuming that people with growth mindset are more likely to actively prepare for the test. While some studies have already explored the association between implicit theories of intelligence and academic achievement, the present study enables a more precise estimation of the strength of the association due to the large sample size. Apart from studying the association between mindset and results in a scholastic aptitude test, we explored whether people with growth mindset are more likely to take an opportunity to participate in a higher number of administrations of the test, hypothesizing that they may be more likely to believe that they could improve between the administrations. Finally, we also explored whether growth mindset predicts improvement between two administrations of the test. 


\section{Method}

\section{Participants}

The materials were administered as a part of a voluntary questionnaire given before administration of the General academic prerequisites (GAP) test used for university admissions in the Czech Republic. The questionnaire with the study materials was administered on a paper right before the GAP test. The questionnaire was given to 6,879 people out of whom 5,989 filled it $(87.1 \%)$. The participants who filled the questionnaire were less likely to be men, $t(6818)=-$ 7.73, $p<.001, d=-0.28,95 \% \mathrm{Cl}=[-0.35,-0.21], M_{\text {filled }}=0.40, M_{\text {did not fill }}=0.54$, were somewhat younger, $t(6877)=-2.69, p=.007, d=-0.10,95 \% \mathrm{Cl}=[-0.17,-0.03], M_{\text {filled }}=20.01, M_{\text {did not fill }}=$ 20.29, but their GAP test results did not differ from the participants who did not fill the questionnaire, $t(6877)=0.75, p=.45, d=0.03,95 \% \mathrm{Cl}=[-0.04,0.10], M_{\text {filled }}=0.00, M_{\text {did not fill }}=-$ 0.02 .

Three hundred and thirty-six participants (5.6\%) failed to properly fill at least one of the measures used in the present study and they were therefore excluded from analysis. The excluded participants had somewhat worse GAP test results than participants who remained in the data set, $t(5987)=-2.68, p=.007, d=-0.15,95 \% \mathrm{Cl}=[-0.26,-0.04], M_{\text {excluded }}=-0.14$, $M_{\text {remaining }}=0.01 .{ }^{1}$ The analysis was performed with data from the remaining 5,653 participants, out of whom $59.6 \%$ were women, $39.7 \%$ were men, and the remaining $0.6 \%$ did not indicate their gender. Most of the participants were 18-20 years old $\left(88.2 \% ; M d n_{\text {age }}=19.3, I Q R_{\text {age }}=0.9\right)$.

Apart from the first questionnaire, we had data available from later administrations of the GAP test, which took place three, seven, and eleven weeks after the first data collection. All administrations took place during the high school academic year. The later administrations were attended by 6,$798 ; 5,427$; and 2616 people respectively. Out of the 5,653 participants from the first data collection, 2,805; 1,782; and 916 participated in later data collections. A mindset measure was only part of the first data collection.

\footnotetext{
${ }^{1}$ Given that the data were not missing completely at random (Schafer \& Graham, 2002), we checked the main results of the reported analyses using data multiply imputed with mice R package (van Buuren \& Groothuis-Oudshoorn, 2011). The results were virtually the same. We therefore report analyses using listwise deletion for simplicity and the results using imputed data can be found at https://osf.io/dk5ab/. Note that it is possible that the data could have been missing not at random if the rate of missing values for mindset items was influenced by mindset itself. This would result in biased estimates of the association between GAP test results and mindset and it should be taken into account when interpreting the results. We do not have any theoretical reason to believe that the missingness was influenced by mindset and the bias would probably not be strong given the relatively low rate of missing data.
} 


\section{Measures}

\section{Mindset}

Participants' mindset was measured using a Czech translation of a scale with two items: "I can learn new things, but I can't really change my basic intelligence" and "I have a certain amount of intelligence and I really can't do much to change it" (Paunesku et al., 2015). The items were introduced by the question: "To what degree do you agree with these statements?" and were rated on a 7-point scale ranging from 1 (strongly agree) to 7 (strongly disagree). Higher ratings therefore indicate a growth mindset. Answers to the two items correlated highly, $r(5651)=.52$, $95 \% \mathrm{Cl}=[.50, .54], p<.001$.

\section{Test of general academic prerequisites}

The GAP test is used for university admissions at the undergraduate level by a wide range of Czech universities with a variety of study programs. Its structure is similar to the Graduate record examination (GRE) used in tertiary education in the United States. It has a pen-andpaper format and it consists of four parts - verbal, quantitative, logic, and argumentation. Each part consists of 22 or 23 items, and is administered separately. Participants have 20 minutes for completion of the verbal part and 30 minutes for each of the three remaining parts. All items have multiple-choice format with 5 possible answers out of which only one is correct. There is no penalty for incorrect answers. Our dataset contained information about test results in the form of a participant's percentile rank in each part of the test and overall result computed as the mean of these percentile ranks. We used McCall transformation (McCall, 1939) for normalization of the scores in all parts of the GAP test as well as for the overall score. That is, we computed percentiles from the variables and then assigned z-scores to the percentiles based on the standard normal distribution. The analysis was conducted using the normalized scores.

The GAP test is administered in total 6 times per academic year and every person can sign up for any number of the administrations. Only the best test result is used by universities for admission decisions. The data used in the present study are from the third to sixth administration in the academic year of 2015/16. We also had available data about the number of administrations for which the participants signed up and data about the number of previous tests they had already taken before the third administration.

The available information about the GAP test shows reasonable psychometric properties. A study with 108 participants conducted using a previous version of the test showed a correlation of .76 of the overall score in the GAP test with the score in the Scholastic aptitude test (SAT; https://osf.io/32rpu/). The same study found Cronbach's $\alpha$ of .91 for the GAP test. Another study with 290 participants showed that the overall score in the GAP test correlates with $r=.5$ with the score in Raven's advanced progressive matrices (https://osf.io/xaeu6/). The GAP test results of the third administration correlated highly with the result of fourth, $r(2803)=.82,95 \% \mathrm{Cl}=[.80$, $.83], p<.001$, fifth $, r(1780)=.82,95 \% \mathrm{Cl}=[.80, .83], p<.001$, and sixth administration, $r(914)$ 
$=.78,95 \% \mathrm{Cl}=[.76, .81], p<.001$, which shows high test-retest reliability of the test. Further information about the test can be found on https://osf.io/gd7ws/.

\section{Results $^{2}$}

The average of the two questions used for measuring mindset $(M=3.97, S D=1.44)$ correlated slightly negatively with the result in the GAP test, $r(5651)=-.03,95 \% \mathrm{Cl}=[-.05,-.00], p=.04$, meaning that participants with more fixed mindset had slightly better results. An analysis conducted using only participants who took part in the test for the first time showed similar results, $r(3220)=-.01,95 \% \mathrm{Cl}=[-.05, .02], p=.41$. The analysis of the two items separately shows that the item "I can learn new things, but I can't really change my basic intelligence." had no relationship to the result in the test, $r(5651)=-.00,95 \% \mathrm{Cl}=[-.03, .02], p=.91$, and the item "I have a certain amount of intelligence and I really can't do much to change it." had a significantly negative, but very weak, relationship to the result, $r(5651)=-.05,95 \% \mathrm{Cl}=[-.07,-$ .02], $p<.001$. The association between the overall results in the GAP test with the two items measuring mindset as well as with their average rating is shown in Figure 1 . It is possible to see that the relationship is not linear and that participants using ratings in the middle of the scale generally performed worse in the GAP test. A polynomial regression using a centered mindset score as a predictor showed that the GAP test results were significantly associated with both mindset score, $t(5650)=-2.41, p=.02, b=-0.022,95 \% \mathrm{Cl}=[-0.040,-0.004]$, and squared mindset score, $t(5650)=7.32, p<.001, b=0.041,95 \% \mathrm{Cl}=[0.030,0.052]$. When the four parts of the GAP test were analyzed separately, the results showed that they all had almost no association with mindset, $-.035<r s(5651)<.009$.

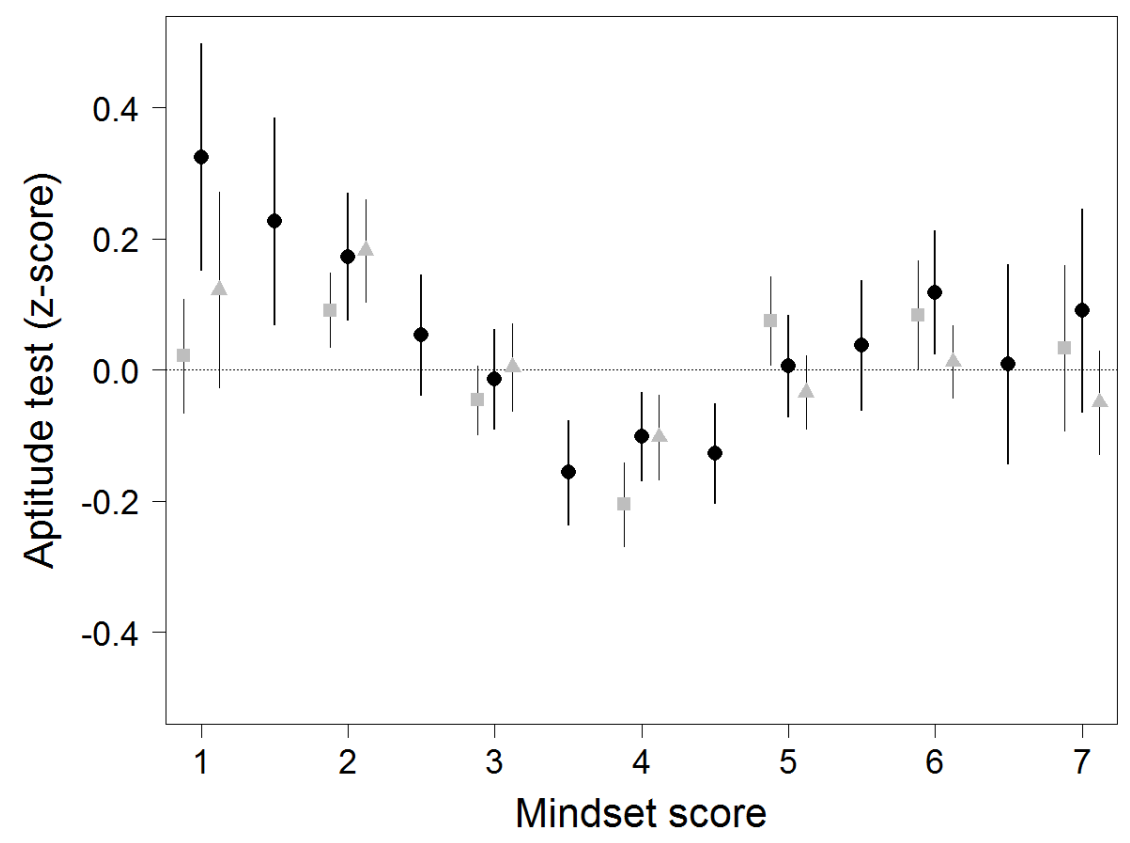

${ }^{2}$ Data and R scripts used for analysis can be found on https://osf.io/vujtd/. 
Figure 1: Association of results in the GAP test with mindset. The figure shows the relationship between results in the GAP test and mindset for the average mindset scores (black circles) and for separate scores for the two items used for measuring mindset (grey). The "I can learn new things" item is shown as a square with a left offset and the item "I have a certain amount of intelligence" as a triangle with a right offset. The error bars represent $95 \%$ confidence intervals of the means. Lower scores correspond to a more fixed mindset.

Some previous research (e.g., Paunesku et al., 2015) suggests that mindset may have an effect on achievement mostly for those whose achievement is generally lower. This means that the relationship between mindset and the GAP test results could differ depending on the performance on the test. The relationship is shown in Figure 2. A possibility of an existence of a breakpoint in the relationship (i.e., a change of a regression slope) was tested using Davies' test (Muggeo, 2008), which did not show a significant change in the regression slope anywhere across the range of the GAP results, $p=.09$.

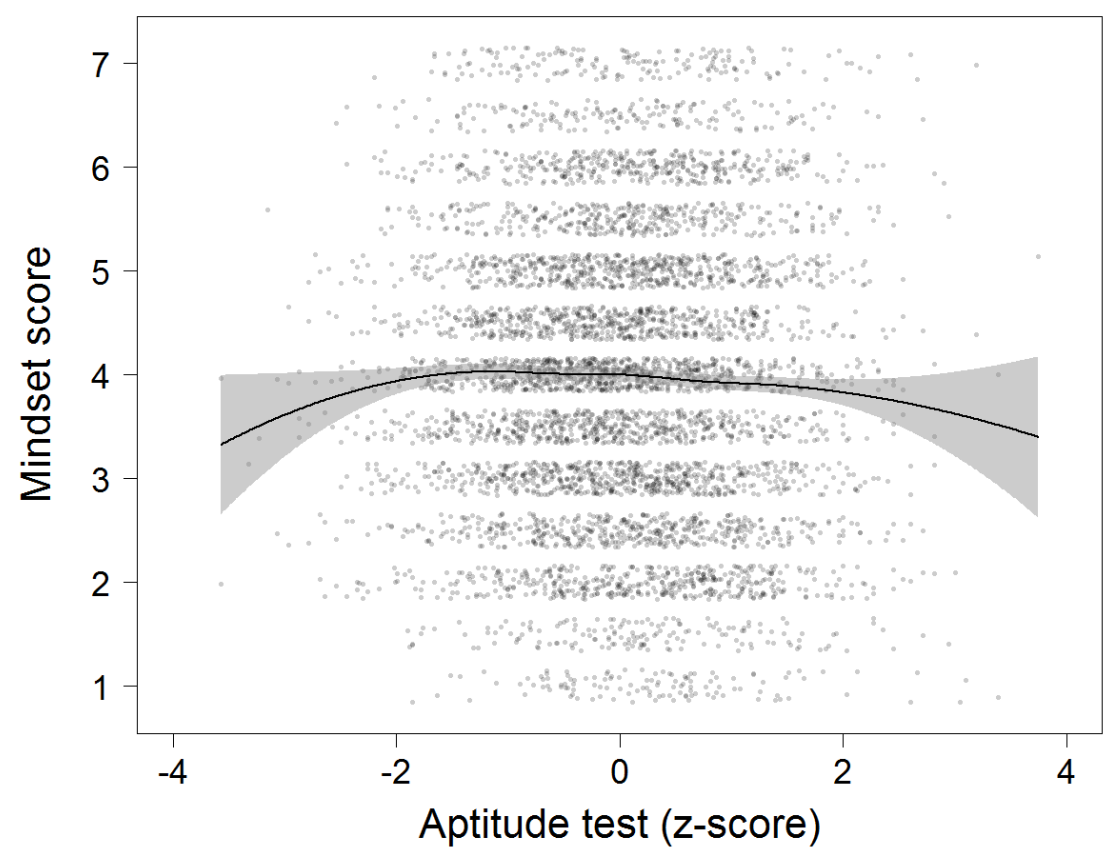

Figure 2: Association of mindset with results in the GAP test. The figure displays the relationship between mindset and results in the GAP test. The black line shows a Loess curve. The shaded region shows a 95\% confidence interval around the Loess curve. Note that we applied jitter to the mindset scores.

Mindset had also no association with the number of GAP tests participants had already taken, $r(5651)=-.02,95 \% \mathrm{Cl}=[-.04, .01], p=.19$, and with the total number of administrations participants signed up for, $r(5651)=-.00,95 \% \mathrm{Cl}=[-.03, .02], p=.90$. We also estimated the association between mindset and the probability of participating in later administrations of the GAP test. The analysis showed that mindset did not predict participation in the fourth administration, $z=0.34, p=.73, O R=1.01,95 \% \mathrm{Cl}=[0.97,1.04]$. The results in the GAP test during the third administration also did not predict later participation, $z=0.58, p=.56, O R=$ $1.05,95 \% \mathrm{Cl}=[0.90,1.22]$, and there was no effect of the interaction of mindset with results in 
the GAP test on participating in the later administration of the GAP test, $z=-1.19, p=.24$, ratio of $O R=0.98,95 \% \mathrm{Cl}=[0.94,1.01]$. Analyses of the association between mindset and participation in fifth and sixth administrations yielded the same results.

Finally, we tested whether mindset was associated with improvement between two administrations using a moderated multiple regression. While the results at the point of the first data collection clearly predicted the results in the fourth administration, $t(2641)=25.80, p<$ $.001, b=0.86,95 \% \mathrm{Cl}=[0.80,0.93]$, we found no association with mindset, $t(2641)=-1.60, p=$ $.11, b=-0.01,95 \% \mathrm{Cl}=[-0.03,0.00]$, as well as no interaction between mindset and the results from the first data collection, $t(2641)=-0.87, p=.38, b=-0.01,95 \% \mathrm{Cl}=[-0.02,0.01]$. The results were virtually the same for fifth and sixth administration of the test. Even for the sixth administration, which took place eleven weeks after the first data collection, mindset did not predict the test results, $t(826)=-1.25, p=.21, b=-0.02,95 \% \mathrm{Cl}=[-0.04,0.01]$.

\section{Discussion}

In the present study we showed that growth mindset is not positively associated with the results in the GAP test. Given that preparation courses for the test exist and free preparation materials and practice tests are also available and coaching was shown to affect results in a similar aptitude test (Becker, 1990; Montgomery \& Lilly, 2012; Powers \& Rock, 1999), we hypothesized that mindset might be associated with improvement in the test. However, we found that mindset did not predict change of the test results between administrations. Moreover, mindset did not predict participation in a future administration of the test or the total number of tests taken by a participant.

Even though we observed an inverted- $U$ shaped relationship between mindset and test results, it is not clear to what degree it can be attributed to a non-linear effect of mindset and to what degree it is a response artefact. That is, participants with lower cognitive abilities might be more likely to use answers near the midpoint of the scale (see e.g., Austin, Deary, \& Egan, 2006; Raaijmakers, van Hoof, 't Hart, Verbogt, \& Vollebergh, 2000).

The lack of positive association between growth mindset and the GAP test results is in contrast to the result of a recent meta-analysis which found that growth mindset is positively, albeit weakly, associated with goal achievement (Burnette et al., 2013). Even though the present study has a relatively large sample size in comparison to previous studies on the topic, it does not invalidate the result of the meta-analysis, which shows large heterogeneity in the association between mindset and goal achievement. When we used the p-curve (Simonsohn, Nelson, \& Simmons, 2014a, 2014b) to evaluate evidential value contained in the studies included in the meta-analysis, we found that studies showing a statistically significant positive effect of growth mindset on goal achievement contain evidential value (see https://osf.io/cd63h/). This suggests that, at least under some conditions, growth mindset is associated with goal achievement. This is supported by the largest study on the topic to this date, which found a positive association between growth mindset and language and math test scores in Chilean high school students (Claro, Paunesku, \& Dweck, 2016). Nevertheless, the 
effect is not present or is in the opposite direction under different circumstances, such as in the present study. Assuming that the U-shaped relationship between mindset and achievement found in the present study occurs also in other domains, a possible explanation of the variance in results might be that participants in some studies tend to have above-average mindset and are therefore predominantly on the part of the mindset scale where the association between mindset and achievement is positive. In other studies, such as the present one, the average mindset is at or below the midpoint of the scale and the association between mindset and achievement is thus absent or even in the opposite direction. Identifying moderators of the effect is an important topic for future research.

We also expected, but did not observe, an association between mindset and change of GAP test results. It is possible that mindset does not influence the amount of preparation for the test and that this caused the lack of the association. However, it is also possible that participants could not have improved much given a relatively short time between two subsequent administrations of the test - the first and the last administration of the test included in the present study were only eleven weeks apart. Consistent with this explanation is a high testretest reliability of the GAP test results observed in the present study, which points out only a limited potential for change.

Apart from testing the association between mindset and results in the GAP test, we also analysed the relationship between mindset and the number of administrations of the GAP test for which participants signed up. We hypothesized that participants who view intelligence as fixed could be less likely to try the test multiple times because they should be more likely to believe that they cannot improve their initial result (Burnette et al., 2013). However, the results did not support this hypothesis. It is important to note that the data about the number of administrations the participants signed up for have some possible limitations. The number of administrations applicants participate in may be dependent, for example, on applicants' financial resources, their motivation, and other factors, which may obscure a possible association with mindset.

Given that we had data from the third administration of the test, it is possible that the lack of association of mindset with results of the GAP test might have been caused by self-selection of those who had worse results in previous administrations. However, this does not seem very likely as we did not find an association between mindset and participation in later administrations of the test. Furthermore, when we analyzed the data only for participants who took part in the test for the first time, we still found no association of the GAP test results with mindset.

Another possible reason why we did not find any relation between mindset and the results of the GAP test is that participants with a growth mindset could have been more willing to apply to a university even if they did not have a high chance of being admitted. That is, given the same abilities, people with a growth mindset might have been more likely to apply to a university and take the GAP test. Previous research suggests that people with a growth mindset are more likely to seek challenges (e.g., Romero, Master, Paunesku, Dweck, \& Gross, 2014; Yeager et al., 2016a) which lends some credence to this hypothesis. Assuming that the effect of mindset 
on self-selection is lower for participants with higher scholastic aptitude, the effect of selfselection should be especially pronounced for participants with worse results in the GAP test and should be barely present in participants with top results since these participants probably know that their chances of being admitted are high even when they have a fixed mindset (Ehrlinger, Mitchum, \& Dweck, 2016). This would in effect lead to a negative relationship between mindset and the GAP test results among participants scoring lowest in the GAP test, which would mask the presumed general positive relationship between growth mindset and the GAP test results. However, the results of the present study show that if there is any positive relationship of growth mindset with the results in the GAP test, it is only for the participants who score lowest in the GAP test, which speaks against this self-selection explanation (see Figure 2). This opposite association would have been, however, predicted by accounts arguing that growth mindset is especially helpful for people who are somehow disadvantaged or have poor abilities (Burnette et al., 2013; Yeager et al., 2014). While the present data are more consistent with this account than with the possibility that growth mindset is generally associated with scholastic aptitude, the statistical test examining a change of the relationship between mindset and results in the GAP test somewhere along the range of the GAP test scores was not significant. Furthermore, this account would still have to explain why the relationship is negative for those with higher scholastic aptitude. A related explanation of our results could argue that people applying for a university are already a sample with above-average abilities and the association with mindset is therefore not present because there were no participants in our study who could have benefited from a growth mindset. People who are already high in scholastic aptitude might also have less room to improve between the administrations. The range restriction of scholastic aptitude in our sample also means that any association between mindset and scholastic aptitude present in the population is likely to be underestimated (Sackett \& Yang, 2000). While this range-restriction account cannot be ruled out based on our results, it would pose a serious limitation to the applicability of the mindset theory.

In sum, we found that mindset had virtually no association with results in a scholastic aptitude test used for university admissions. While the association between mindset and goal achievement was previously shown to be weak (Burnette et al., 2013), our study presents a large amount of new data suggesting that the association may be even weaker than previously thought. Given that recent large scale experiments suggest that learning growth mindset improves academic achievement (Paunesku et al., 2015; Yeager et al., 2016a, b), our study does not invalidate the notion that implicit theories of intelligence might be a promising target for educational interventions. However, it suggests that mindset might not be as useful for predicting future success or that its predictive abilities are at least limited to specific circumstances. Yet, we note that our study has several limitations including possible selfselection and range-restriction effects, a short measure of mindset, and a short duration between subsequent administrations of the test. We also did not include measures of hypothesized mediating variables, such as the amount of practice, and the mindset measure was not directly tailored to assess beliefs about the possibility of improvement in the GAP test. Future studies may overcome these limitations and thus better explain differences between results of the present and past studies. 


\section{Acknowledgements}

The work of Štěpán Bahník was supported by Internal Grant Agency of Faculty of Business Administration, University of Economics, Prague (IP300040). The work of Marek Vranka was supported by Charles University, Prague (PRVOUK7 and PRVOUK15). We would like to thank Scio company for the data from the National Comparative Exams GAP test used in the study.

\section{References}

Austin, E. J., Deary, I. J., \& Egan, V. (2006). Individual differences in response scale use: Mixed Rasch modelling of responses to NEO-FFI items. Personality and Individual Differences, 40, 1235-1245.

Becker, B. J. (1990). Coaching for the Scholastic Aptitude Test: Further synthesis and appraisal. Review of Educational Research, 60, 373-417.

Blackwell, L. S., Trzesniewski, K. H., \& Dweck, C. S. (2007). Implicit theories of intelligence predict achievement across an adolescent transition: A longitudinal study and an intervention. Child development, 78, 246-263.

Burnette, J. L., O'Boyle, E. H., VanEpps, E. M., Pollack, J. M., \& Finkel, E. J. (2013). Mind-sets matter: A meta-analytic review of implicit theories and self-regulation. Psychological Bulletin, 139, 655.

Claro, S., Paunesku, D., \& Dweck, C. S. (2016). Growth mindset tempers the effects of poverty on academic achievement. Proceedings of the National Academy of Sciences, 113, 86648668.

Dweck, C. S. (2012). Mindset: How you can fulfil your potential. Hachette UK.

Dweck, C. S., \& Leggett, E. L. (1988). A social-cognitive approach to motivation and personality. Psychological review, 95, 256-273.

Ehrlinger, J., Mitchum, A. L., \& Dweck, C. S. (2016). Understanding overconfidence: Theories of intelligence, preferential attention, and distorted self-assessment. Journal of Experimental Social Psychology, 63, 94-100.

McCall, W. A. (1939). Measurement: A Revision of How to Measure Education. New York, NY: Macmillan.

Molden, D. C., \& Dweck, C. S. (2006). Finding" meaning" in psychology: a lay theories approach to self-regulation, social perception, and social development.American Psychologist, 61, 192-203. 
Montgomery, P., \& Lilly, J. (2012). Systematic reviews of the effects of preparatory courses on university entrance examinations in high school-age students. International Journal of Social Welfare, 21, 3-12.

Muggeo, V. (2008) Segmented: an R package to fit regression models with broken-line relationships. $R$ News, 8, 1: 20-25.

Neisser, U., Boodoo, G., Bouchard, T. J., Jr., Boykin, A. W., Brody, N., Ceci, S. J., ... \& Urbina, S. (1996). Intelligence: knowns and unknowns. American psychologist, 51, 77-101.

Paunesku, D., Walton, G. M., Romero, C., Smith, E. N., Yeager, D. S., \& Dweck, C. S. (2015). Mind-set interventions are a scalable treatment for academic underachievement. Psychological science, 26, 784-793.

Powers, D. E. (1985). Effects of Test Preparation on the Validity of a of a Graduate Admissions Test. Applied Psychological Measurement, 9, 179-190.

Powers, D. E., \& Rock, D. A. (1999). Effects of Coaching on SAT I: Reasoning Test Scores. Journal of Educational Measurement, 36, 93-118.

Raaijmakers, Q. A. W., Van Hoof, A., 't Hart, H., Verbogt, T. F. M. A., \& Vollebergh, W. A. M. (2000). Adolescents' midpoint responses on Likert-type scale items: Neutral or missing values. International Journal of Public Opinion Research, 12, 208-216.

Rattan, A., Savani, K., Chugh, D., \& Dweck, C. S. (2015). Leveraging Mindsets to Promote Academic Achievement Policy Recommendations. Perspectives on Psychological Science, 10, 721-726.

Romero, C., Master, A., Paunesku, D., Dweck, C. S., \& Gross, J. J. (2014). Academic and emotional functioning in middle school: The role of implicit theories. Emotion, 14, 227-234.

Sackett, P. R., \& Yang, H. (2000). Correction for range restriction: An expanded typology. Journal of Applied Psychology, 85, 112-118.

Schafer, J. L., \& Graham, J. W. (2002). Missing data: Our view of the state of the art. Psychological Methods, 7, 147-177.

Simonsohn, U., Nelson, L. D., \& Simmons, J. P. (2014a). P-curve and effect size correcting for publication bias using only significant results. Perspectives on Psychological Science, 9 , 666-681.

Simonsohn, U., Nelson, L. D., \& Simmons, J. P. (2014b). P-curve: A key to the file-drawer. Journal of Experimental Psychology: General, 143, 534-547.

van Buuren S., \& Groothuis-Oudshoorn K. (2011). mice: Multivariate Imputation by Chained Equations in R. Journal of Statistical Software, 45, 1-67.

Yeager, D. S., Johnson, R., Spitzer, B. J., Trzesniewski, K. H., Powers, J., \& Dweck, C. S. (2014). The far-reaching effects of believing people can change: Implicit theories of 
personality shape stress, health, and achievement during adolescence. Journal of Personality and Social Psychology, 106, 867-884.

Yeager, D. S., Romero, C., Paunesku, D., Hulleman, C. S., Schneider, B., Hinojosa, C., ... \& Trott, J. (2016a). Using design thinking to improve psychological interventions: The case of the growth mindset during the transition to high school. Journal of Educational Psychology, $108,374-391$.

Yeager, D. S., Walton, G. M., Brady, S. T., Akcinar, E. N., Paunesku, D., Keane, L., ... \& Gomez, E. M. (2016b). Teaching a lay theory before college narrows achievement gaps at scale. Proceedings of the National Academy of Sciences, 113, E3341-E3348. 